\title{
An Architectural Approach to Provide Efficient Service with The Service Level Agreement
}

\author{
${ }^{*}$ N. Bhargavi ${ }^{1}$, Dr. R. Raja Sekhar ${ }^{2}$ \\ ${ }^{1}$ M.Tech Student, Department of CSE, JNTUA, Ananthapuramu, Andhra Pradesh, India \\ ${ }^{2}$ Associate Professor, Department of CSE, JNTUA, Ananthapuramu, Andhra Pradesh, India
}

\section{Article Info}

Volume 7, Issue 1

Page Number: 161-166

Publication Issue :

January-February-2021

\section{Article History}

Accepted : 01 Feb 2021

Published : 08 Feb 2021

\section{ABSTRACT}

Cloud brokers have been as of late presented as an extra computational layer to encourage cloud choice and administration the board assignments for cloud shoppers. In any case, existing business plans on cloud administration choice ordinarily expect that brokers are totally trusted, and don't give any assurance over the accuracy of the administration suggestions. It is then workable for an undermined or untrustworthy intermediary to effortlessly exploit the restricted abilities of the customers and give off base or deficient reactions. To address this issue, we propose a creative Cloud Service Selection Verification (CSSV) conspire and list structures to empower cloud customers to distinguish misconduct of cloud dealers during the administration determination measure. We show rightness and productivity of our methodologies both hypothetically and observationally.

Keywords: Cloud Service Selection, Brokerage System

\section{INTRODUCTION}

I. Presentation this has brought about an enormous number of cloud administration suppliers (CSPs), offering a wide scope of assets. The accessibility of different, potentially complex choices, be that as it may, makes it hard for potential cloud customers to gauge and choose which choices suit their prerequisites the best. The challenges are twofold: 1) It is hard for cloud customers to assemble data pretty much all the CSPs accessible for their choices; 2) It is additionally computationally costly to pick a reasonable CSP from a possibly enormous CSP pool. Considering

these troubles, both industry and the scholarly world (see for a review) proposed acquainting an extra processing layer(referred with as cloud business systems)on top of the base assistance provisioning to empower errands, for example, revelation, intervention and checking. In a cloud business framework, one of the most basic assignments is to give excellent choice administrations to customers. That is, a agent gives customers a rundown of suggested CSPs that address the customers' issues. With the guide of cloud intermediaries, customers at this point don't have to gather, search or think about CSPs' administrations furthermore, capacities. Without the capacity to check the rightness of the administration proposal, cloud customers could be without any problem cheated by vindictive merchants. For example, noxious agents could suggest their good CSPs however much as could be expected what's more, disregard other reasonable CSPs, 
without being gotten by the customers. All the more truly, because of the absence of super vision and confirmation of intermediaries' activities, vindictive agents could even suggest malignant CSPs which gather and sell customers' private assets, screen customers' hosts during cloud administration provisioning, causing major money related and privacy misfortunes to the customers. Hence, it is critical to prepare the customers with check abilities of the got proposals. The customers should not check each proposalresult;however, they unquestionably need to have the capacity to do so when they feel important.

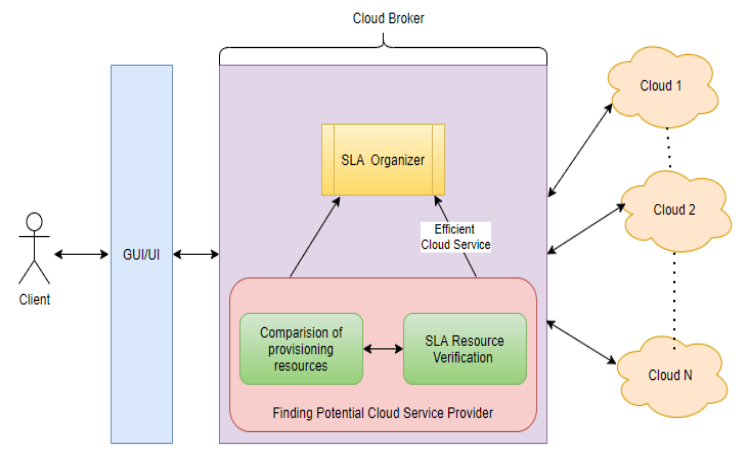

Fig 1: Architecture

\section{RELATED WORKS}

Cloud delegates are as of late introduced as an extra computational layer to support cloud decision likewise, organization the board tasks for cloud buyers. Be that since it might, existing lender plans on cloud organization decision routinely expect that middle people are completely reliable and do not provide any assurance over company proposition correctness. Further, it has been functional aimed at corrupt or haggled mediatorfor exploiting successfully the confined of buyers and provides inadequacy or mistaken responses. Forhandling this issue. Weight changing has been broadly focused inside the composition, and different computations are proposed. Many analysis efforts have conjointly been devoted into cloud brokerage [1], which project distinct types of brokerage architecture, accumulation of service, sharing of resource, and assignment and many more. The work [2] addresses other criterion of selection that intents to maximal the survival of information probability or count of information surviving. They project active programming algorithm depending on wellrecognized knapsack issue.Makers in [3] projected a 2 phase procedure for enhancing the task booking show and lessening the task inconsequential task allocation in the fogs. The Bayes classifier rule is first gotten for requesting tasks snared in to legitimate arranging data, and a while later endeavors are logically coordinated with relating concrete VMs. In any case, the Bayes classifier essentials critical chronicled data to encourage precise results, \& accordingly, this framework prompts precision disaster. The investigation uncovered in [4] introduced a prerequisite based task scheduler that positions customers upheld task length and memory. A cross variety improvement estimation that accompanies innate and PSO counts for e_cient task booking was moreover projected. A line boss has been utilized for storing and organizing out customer endeavors. Excused tasks from the coordinated line have beenspecified to on-demand line, \& endeavors from the 2 lines are specified to combination figuring. Taking everything into account, slow gathering rate and vulnerable area search limit are seen from the PSO computation. In [6], a blend smoothing out estimation was suggested that solidifies cuckoo and friendliness examine figuring's to make up a shrewd technique for improving the plan cycle. Endeavors are at first apportioned towards VMs as well as known confines that approve the utilization of energy, memory, discipline and credits have been solved. The researchers in contribution [7] projected active voltage and recurrent scaling, which represents requirements of company with minimal repetition. The utilization of scope of energy has been utilized and assessed for settling other frequencies. The runtime enhancement has been examined further and movement of task and modifying the repeated plots have specified for lessening the employee 
energy utilization. Huge of projected heterogeneous solid SJF approach for real-central approach and huge architectural time has been consumed.

Energy usage is lessened by strategies for dynamic provisioning and so the dynamic requesting is expedited with dynamic heterogeneity through this fashion decreasing create span and increasing resources utility.The dynamic heterogeneities of residual job that need be done what's more, resources are throughout about. Dynamic sales for heterogeneous resources are portrayed to limit execution time and augmentation resource use. intended to handle the trouble of dynamic weight changing by proposing a mutt PSO estimation. Remaining weight is seized across. di_erent resources by methods for trouble changing. The different digit work maintain vector machine (SVM) estimation is used to survey the disrupting impacts in exploitation numerous resources. A consolidated cloud-based media structure that contains a resource boss, pack heads and specialist bunches is accepted. Client requests are distributed by the resource boss to the laborers within its bundle head. Regardless the look amount of SVM computation is moderate. Reference[9] introduced the cutoff time careful need arranging model, which restricts the conventional makespan and lifts resource use under cutoff time necessity. Endeavors are anticipated climbing demand snared in to length need and facilitated to the sensible VM with least taking care of time. Reference [5] proposed the weakness careful web booking computation for overhauling the display of cloud organization stages. The count of endeavors that direct post for VM's is controlled, proactive and responsive methods are merged. The component limits of employments are noncommissioned when they are submitted and tasks are allotted to assist cases for execution. Reference [8] improved the energy e-ciency of heterogeneous specialists throughout a sent registering structure by ways for a noncooperative game-based model. The model addresses working utility limit as unit power e-ciency and shows the presence of the nash balance motivation behind game. totally the sales entirety inside the system fills in as a necessity, and in this manner the reasonable task sum is picked by each specialist that matches it with the ideal resource for influence customer interest. Reference [10] gave a cutoff time careful PSO computation for overhauling the introduction of task booking counts. Two limits, particularly cutoff time and advantage, are utilized by the figuring and resolved to describe endeavors. Each endeavor is reserved to VMs upheld need using the PSO figuring. If two tasks have an indistinguishable cutoff time regards, around then the premier outrageous customer pay regard, which cares cutoff time and million bearings, is figured. Endeavors are reserved upheld worth. In any case, the PSO computation shows low intermixing time.

\section{PROPOSED APPROACH}

In this section, we project a CSSV (cloud-selectionservice verification) devise and architectures of file for employing the clients of cloud for discriminating the cloud-dealers mischief at the time of selection of administration cycle. One need to exhibit and effectively of our techniques both precisely and hypothetically has been happened in below fig. 1

Proposed another presentation estimating strategy for Framework As-Service contributions, considering the sort of administrations running in a virtual machine. Introduced a system for observing cloud execution dependent on clients' criticism. Li and Wang moreover proposed a likelihood technique to assess the emotional reliability of the administration segment just as the entire composite administration from a progression of appraisals given by clients. The range tree-based technique proposed in requirements to fabricate and install a Merkle hash tree for every hub, and. this cycle is likewise recursively conjured for the hubs of the installed Merkle hash tree, which 
makes file construction, questioning also, check amazingly tedious; the VB-tree in is not effective for questions on non-key properties since it will create enormous size evidence messages to cover the hubs in between the question runs however don't contain theinquiry results.

\section{A. Cloud Service Selection}

It is important that, the curiosity of our methodologies not just lies in another arrangement of confirmation calculations explicit to the cloud administration choice, yet in addition gives proficient arrangements (contrasted and the cutting edge) to the issue of verifying multidimensional questions. The motivation to pick Cost as the ordering field is twooverlay. To start with, given that most cloud suppliers utilize a compensation for each utilization plan of action, Price is one of the most regularly happened rules in cloud administration choice questions. To begin with, cloud administration choice normally permits cloud clients to determine numerous assistance necessity is consistently alluring to have productive cloud administration determination and confirmation with the goal that the cloud end clients would not feel deferral of administrations. Our tale file structure is the center part of our Cloud Service Selection Verification (CSSV) conspire, which utilizes the possibility of "partition of obligations" to guarantee solid security ensures. we propose the Cloud Service Choice Verification (CSSV) plot which is a thorough arrangement that is fit for ensuring all the three security necessities (i.e., realness, satisfiability also, culmination).

\section{B. Brokerage System}

In a cloud financier framework, one of the most basic assignments is to give top notch choice administrations to customers. That is, a specialist gives customers a rundown of suggested CSPs that address the customers' issues. With the guide of cloud merchants, customers at this point don't have to gather, search or analyze CSPs' administrations and abilities Precisely, we present a confided in gatherer in the cloud financier framework that isolates the undertaking of CSP data assortment from the administration determination. The authority doesn't legitimately cooperate with the cloud customers and is just accountable for social event data from the CSPs, and thus it very well may be more dedicated into receiving modern safeguards to sift through risky information furthermore, building a validated information base of CSPs' profiles.

\section{Check and Verification Strategy}

All the more truly, because of the absence of oversight and check of representatives' activities, noxious intermediaries could even suggest pernicious CSPs which gather and sell customers' private assets, screen customers' hosts during cloud administration provisioning, causing major monetary and privacy misfortunes to the customers. we propose inventive verified record structures and confirmation conventions to permit customers to check the culmination and validness of representatives' answers. This issue is identified with that of confirmation of inquiry results for re-appropriated information bases. choice and check so that the cloud end clients would not feel deferral of administrations, yet existing hardly any works, despite the fact that help confirmation of multidimensional question results, are tedious, coming about that they couldn't fulfill the needs of the present continuous cloud administration proposals

\section{Algorithmic View}

This algorithmic view discusses about the various informational view of the comparison the cloud service provider.

Prioritized data center algorithm is used for finding an optimized cloud service from a huge pool of services. In this algorithm, based on the priority and by considering some parameters of cloud service, the 
cloud brokerage system recommends an efficient service to the customer.The implementation of the Prioritized Data Center algorithm is described using the following pseudo-code:

\section{Prioritized Data Center Algorithm:}

Input: cloud provider $\mathrm{CP}$,customer priority $\mathrm{t}$, maximum willingness $\mathrm{W}$, minimum availability $\mathrm{A}$, max response time $\mathrm{R}$, minimum throughput $\mathrm{T}$, EligibleList E.

For all Cloud Providers CPDo

If getPriority $(\mathrm{CP})<=\mathrm{W}(\mathrm{t})$

Then add CP to EligibleList

\section{Endif}

If sizeof ( EligibleList $<=1$ )

Then

Return CP

Else

For all EligibleList E DO

Calculate Responsetime(E)

Ifget $\mathrm{A}(\mathrm{E})>=\mathrm{A}(\mathrm{t})$ and get $\mathrm{T}(\mathrm{E})>=\mathrm{T}(\mathrm{t})$

Then add CP toFinalList

\section{Endif}

Endfor

Endif

Endfor

If $\quad$ sizeof (FinalList) $>0$ )

Then

Choose random cloud provider $\mathrm{C}$ from final list

Return C

Else

Return $\mathrm{C}=$ null

\section{Endif}

Output: $\quad$ Optimized cloud providerP

\section{RESULTS}

The following results are obtained from the implementation of the proposed system.

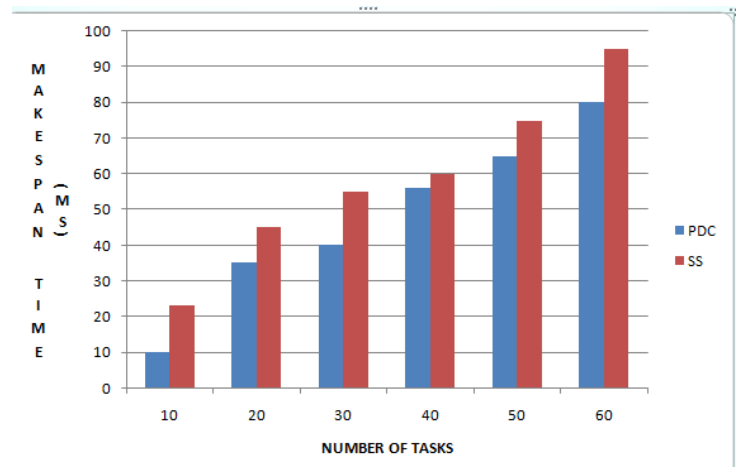

Fig 1 : Makespan Time

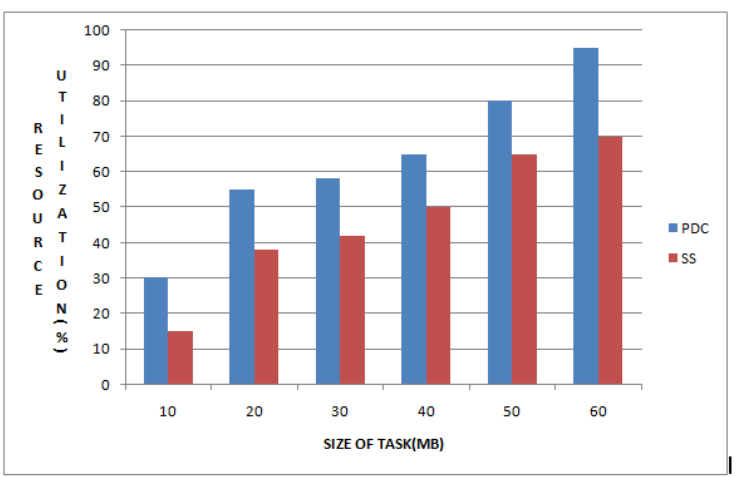

Fig 2 : Resource Utilization

\section{CONCLUSION}

Cloud specialists have been starting late introduced as an extra computational layer to energize cloud decision and organization the board tasks for cloud customers. Regardless, existing strategies on cloud organization decision conventionally expect that dealers have been reliable completely, and does not provide any assurance over company proposals precision. Later, it has been service for dishonest or subverted mediator for simply misusing the confined clients capacities and inadequacy responses. For addressing this problem or challenge, we project CSSV and their architectures have been listed for allowing the consumers of cloud for identifying the unwanted conduct of vendors of cloud at the time of measuring the assurance of an organization. We 
exhibit effectiveness and process of both observationally as well as practically.

\section{REFERENCES}

[1]. S. at TechTarget. New servers: 2011 top cloud computing provider, 2011.

[2]. C. W. Chang, P. Liu, and J. J. Wu. Probabilitybased cloud storage providers selection algorithms with maximum availability. In 41 st International Conference on Parallel processing, 2012.

[3]. Yu, L.; Chen, L.; Cai, Z.; Shen, H.; Liang, Y.; Pan, Y. Stochastic load balancing for virtual resource management in datacenters. IEEE Trans. Cloud Computing. 2016.

[4]. Shen, H. RIAL; Resource intensity aware load balancing in clouds. IEEE Trans. Cloud Computing. 2017.

[5]. Rani, E.; Kaur, H. E_cient Load Balancing Task scheduling in Cloud Computing using Raven Roosting Optimization Algorithm. Int. J. Adv. Res. Computing. Sci. 2017.

[6]. Zhang, P.; Zhou, M. Dynamic cloud task scheduling based on a two-stage strategy. IEEE Trans. Automation Science Eng. 2018, 15, 772783.

[7]. Krishnadoss, P.; Jacob, P. OCSA: task scheduling algorithm in cloud computing environment. Int. J. Intell. Engin. Syst. 2018, 11, 271-279.

[8]. Alworafi, M.A.; Mallappa, S. An enhanced task scheduling in cloud computing based on deadline-awaremodel. Int. Grid High Perform. Comput. 2018, 10, 31-53.

[9]. Polepally, V.; Chatrapati, K. S. Dragonfly optimization and constraint measure-based balancing in cloud computing. Cluster comput. 2017, 1-13.

[10].Fan, G.; Chen, L.; Yu, H.; Liu, D. Modeling and Analyzing Dynamic Fault-Tolerant Strategy for Deadline Constrained Task Scheduling in Cloud
Computing. IEEE Trans. Syst. Man Cybernet. Syst. 2017, 1-15.

\section{Cite this article as :}

N. Bhargavi, Dr. R. Raja Sekhar, "An Architectural Approach to Provide Efficient Service with The Service Level Agreement", International Journal of Scientific Research in Computer Science, Engineering and Information Technology (IJSRCSEIT), ISSN : 2456-3307, Volume 7 Issue 1, pp. 161-166, JanuaryFebruary 2021. Available at doi : https://doi.org/10.32628/CSEIT217133 Journal URL : http://ijsrcseit.com/CSEIT217133 